\title{
Somatostatinoma de papila maior e menor associado a tumor do estroma gastrointestinal (GIST)*
}

\author{
Somatostatinoma of the papilla major and \\ minor associated with gastrointestinal \\ stromal tumor (GIST)
}

\author{
Alberto de Almeida Sartorelli Junior ${ }^{1}$, Jéssica Andrea Bravo \\ Santana ${ }^{2}$, Mariana Fabbri Guazzelli de O. Pereira ${ }^{3}$, \\ José Jukemura ${ }^{4}$, José Eduardo Monteiro da Cunha ${ }^{5}$, \\ Romeu Nunes de Assunção Neto ${ }^{6}$
}

\begin{abstract}
Sartorelli Jr, AA, Santana JAB, Pereira MFGO, Jukemura J, Cunha JEM, Assunção Neto RN. Somatostatinoma de papila maior e menor associado a tumor do estroma gastrointestinal (GIST). Rev Med (São Paulo). 2008 jan.-mar.;87(1):58-65.

RESUMO: Os somatostatinomas são tumores neuroendócrinos extremamente raros que possuem positividade imunohistoquímica para somatostatina. A incidência destes tumores é maior nos pacientes portadores da síndrome de Von Recklinghausen. Existem quatro casos relatados na literatura que mostram coexistência entre somatostatinoma e tumores do estroma gatrointestinal (GIST), todos em pacientes com neurofibromatose. Neste relato, descrevemos uma paciente não portadora da síndrome que apresentou dois somatostatinomas primários de papila maior e menor e nove nódulos jejunais de GIST. O diagnóstico foi confirmado pelo anátomo-patológico e imunohistoquímica, e o tratamento consistiu em uma duodenopancreatectomia associada à ressecção dos nódulos jejunais. A paciente evoluiu com gastroparesia no pós-operatório, e recebeu alta no 34 $\mathrm{PO}$ com aceitação de dieta oral. Este é o primeiro caso de toda a literatura que descreve apresentação simultânea destes tumores em paciente não portadora de neurofibromatose.
\end{abstract}

DESCRITORES: Somatostatinoma. Tumores do estroma gastrointestinal/cirurgia Ampola Hepatopancreática/cirurgia. Imunohistoquímica.

\footnotetext{
* Prêmio Oswaldo Cruz - XXVI COMU, 2007 - Área: Relato de Caso.

1. Acadêmico do $6^{\circ}$ ano da Faculdade de Medicina da USP.

2. Acadêmica do $5^{\circ}$ ano da Faculdade de Medicina da USP.

3. Acadêmica do $3^{\circ}$ ano da Faculdade de Medicina da USP.

4. Orientador. Médico Doutor do Departamento Gastroenterologia da FMUSP.

5. Médico Docente do Departamento de Gastroenterologia da FMUSP.

6. Médico residente do Departamento de Patologia da FMUSP.
}

Endereçopara correspondência: Alberto de Almeida Sartorelli Junior. Rua Ministro Roberto Cardoso Alves, 815. Alto da Boa Vista. São Paulo, SP, CEP: 04737-000. email: albertosartorelli@yahoo.com.br 


\section{INTRODUÇÃO}

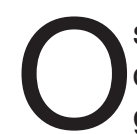
$s$ tumores neuroendócrinos correspondem a $2 \%$ das neoplasias gastrointestinais, sendo que menos de $5 \%$ destes estão localizados no duodeno e papila duodenal $^{6,28}$. O somatostatinoma é o subtipo mais raro; tem sua origem a partir das células $D$ produtoras de somatostatina, sendo o diagnóstico baseado na positividade imunohistoquímica para grânulos citoplasmáticos deste peptídeo ${ }^{16}$. Em apenas 10\% dos casos há liberação excessiva do hormônio na circulação, acarretando uma síndrome caracterizada por diabetes mellitus, colelitíase e diarréia com esteatorréia ${ }^{25,33,44}$.

A coexistência de somatostatinoma com os tumores do estroma gastrointestinal (GIST) é extremamente rara, havendo apenas quatro relatos de caso na literatura, todos em pacientes portadores de neurofibromatose tipo $1^{4,14,36,40}$.

$O$ presente relato de caso consiste em uma paciente não portadora da doença de Von Recklinghausen com dois somatostatinomas primários, nas papilas maior e menor, associados com GIST duodenal. Este é o primeiro relato desta apresentação na literatura.

\section{RELATO DE CASO}

Paciente de 39 anos, negra, do sexo feminino, nascida e residente em São Paulo, iniciou quadro de icterícia obstrutiva acompanhada de forte dor em cólica no hipocôndrio direito, e alguns episódios febris. Investigada em outro serviço, foram pedidos marcadores séricos tumorais, os quais vieram dentro dos limites normais (Tabela 1). Realizaram-se ultrassom e tomografia computadorizada do abdome, os quais constataram colelitíase, dilatação de vias biliares intra e extra-hepática e dilatação do ducto de Wirsung (Figura 1). A paciente também foi submetida à colangiopancreatografia retrógrada endoscópica, obtendo-se como achado abaulamento da papila duodenal maior e menor. Executou-se papilotomia da papila maior e biópsia das duas lesões, cujo resultado da microscopia foi inicialmente sugestivo de adenocarcinoma. Após o exame, evoluiu com colangite, sendo tratada com antibioticoterapia. Neste momento, foi encaminhada para o Serviço de Gastrocirurgia do Hospital das Clínicas e submetida a tratamento cirúrgico. Foi executada duodenopancreatectomia com reconstrução em dupla alça, sendo a peça cirúrgica retirada em monobloco (Figura 2A). No intraoperatório, além dos tumores nas duas papilas, foram constatados nove nódulos em jejuno proximal, dos quais cinco foram ressecados separadamente da peça e enviados para anátomo-patológico (Figura 2B). No pós-operatório, a paciente evoluiu com gastroparesia manifestada por não aceitação de dieta oral. Tratada clinicamente, recebeu alta no $34^{\circ} \mathrm{PO}$.

Tabela 1. Exames laboratoriais e marcadores séricos tumorais no início do quadro

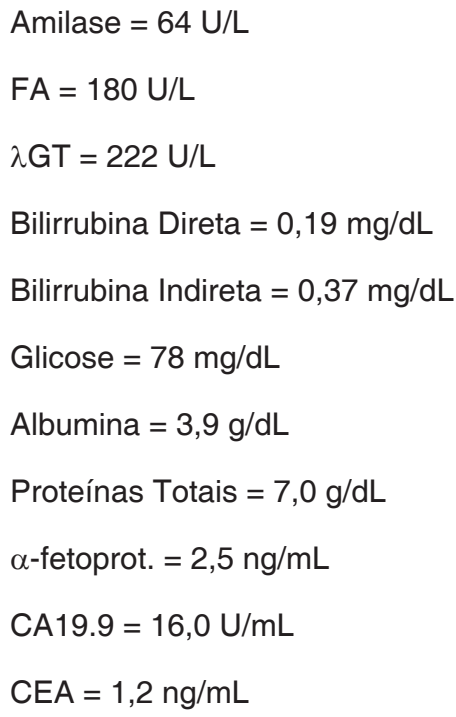

Referência: 28 a 100 U/L

Referência: 35 a 104 U/L

Referência: 5 a 36 U/L

Referência: inferior a 0,30 mg/dL

Referência: 0,10 a 0,60 mg/dL

Referência: 70 a 100 mg/dL

Referência: 3,4 a 4,8 g/dL

Referência: 6,6 a 8,7 g/dL

Referência: até $10,0 \mathrm{ng} / \mathrm{mL}$

Referência: até $37,0 \mathrm{U} / \mathrm{mL}$

Referência: até 5,0 ng/mL 

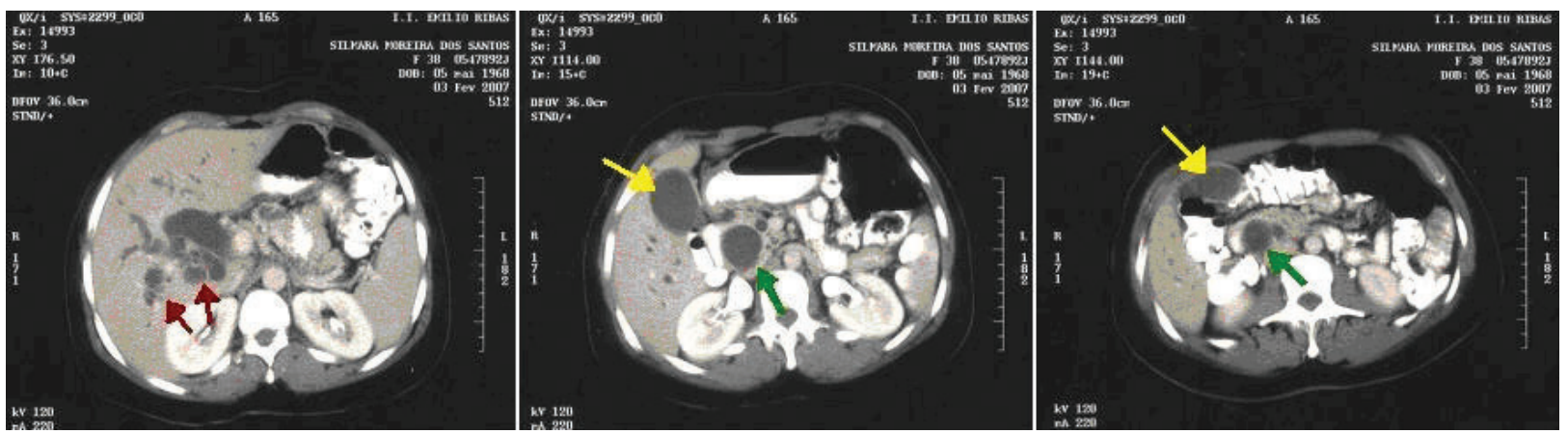

Figura 1. Cortes tomográficos mostrando dilatação de vias biliares intra (seta vermelha) e extra (seta verde) hepática, até o nível da papila. Também se observa vesícula biliar cheia (seta amarela)

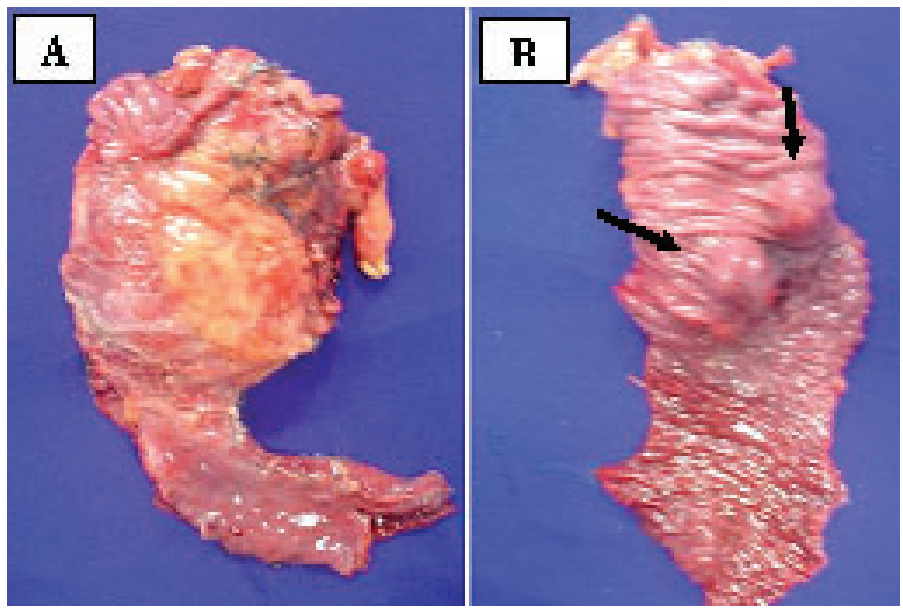

Figura 2. A) Peça cirúrgica resultante da duodenopancreatectomia; B) Dois tumores nas papilas maior e menor

\section{Anátomo-patológico e imunohistoquímica}

Analisaram-se nove nódulos medindo entre 0,1 e $1,0 \mathrm{~cm}$, localizados em parede do jejuno, compatíveis com tumor do estroma gastrointestinal (Figura 3A). Foram também analisadas duas lesões medindo 2,3 e 1,0 cm no maior eixo, distantes 0,8 $\mathrm{cm}$ entre si, localizadas respectivamente nas papilas maior e menor. Ambas as lesões estendiam-se até a camada muscular própria do duodeno. À microscopia, os dois tumores eram constituídos por estruturas tubulares, sólidas e trabeculares, com atipia leve (Figura 3B). As margens pancreática, intestinais e de colédoco mostraram-se livres de neoplasia e foi observada metástase para um linfonodo pancreáticoduodenal, do total de três dissecados.
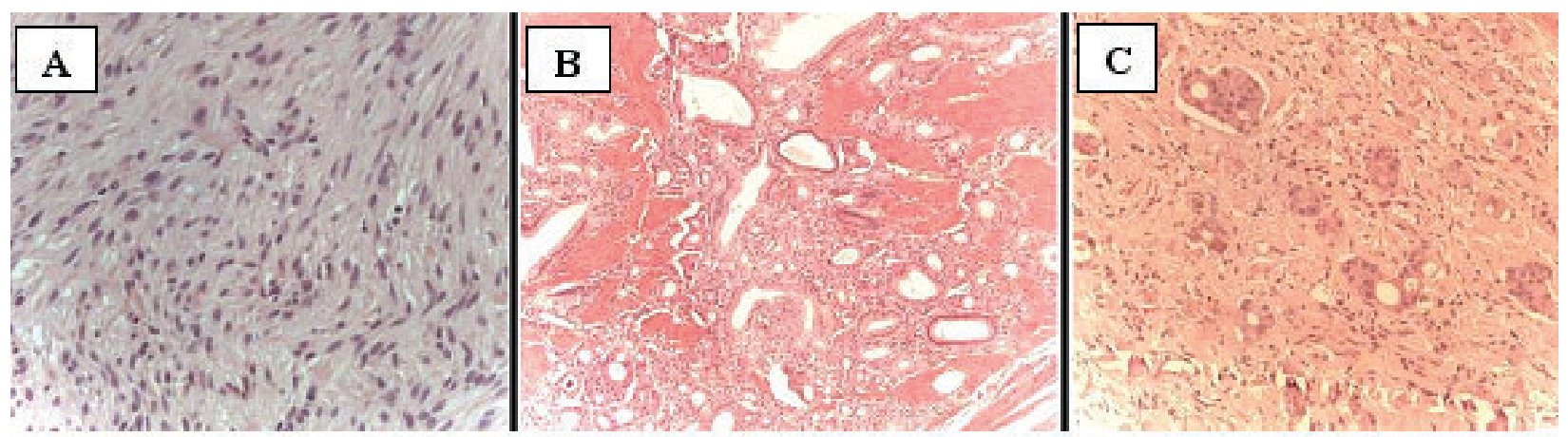

Figura 3. A) Microscopia de um nódulo jejunal, mostrando células alongadas fusocelulares, compatível com GIST. Aumento de 40x; B) Microscopia da lesão na papila maior, mostrando estruturas glandulares envoltas por tecido conjuntivo. Aumento de 20x; C) Microscopia da lesão na papila menor, com as mesmas características da lesão da papila maior. Aumento de 20x 
Na imunohistoquímica, os nódulos de parede jejunal apresentaram positividade global para CD117 (c-kit), fechando diagnóstico para GIST (Figura 4A e
4B). Também foi encontrada positividade focal para actina de músculo liso (Figura 4C), e negatividade para proteína S-100 (Figura 4D).
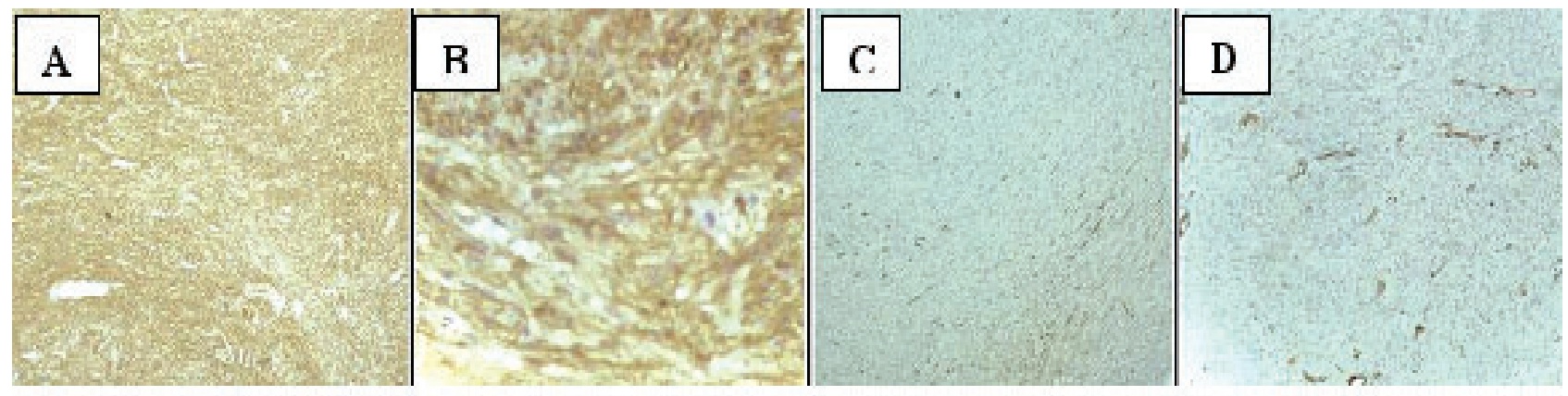

Figura 4. Imunohistoquímica de um dos tumores jejunais, onde se observa positividade global para CD117 (A); com padrão citoplasmático sem corar os núcleos celulares (B); permitindo fechar o diagnóstico para GIST. Pode-se também observar alguma positividade para actina de músculo liso (C); e ausência de coloração para proteína S-100 (D). Os outros nódulos jejunais apresentaram o mesmo padrão imunohistoquímico

Nos dois tumores das papilas, foi encontrada positividade global para cromogranina e sinaptofisina (Figuras 5A e 5B), e positividade em raras células para citoqueratinas $\mathrm{AE}-1 / \mathrm{AE}-3$, sendo este perfil compatível com tumores neuroendócrinos. Observouse positividade em menos de $2 \%$ das células para o marcador de replicação celular Ki67 (Figura 5C), sendo este um achado que corresponde a bom prognóstico $^{45}$. Foi também verificada positividade global para somatostatina (Figura 5D), e negatividade para todos os outros hormônios pancreáticos (Figuras 5E, 5F, 5G, 5H e 5J), possibilitando classificar estes dois tumores em somatostatinomas.

Os dois tumores neuroendócrinos foram considerados como subclasse 2 (carcinomas neuroendócrinos), conforme a classificação criada pela World Health Organization (WHO) em 2000 e revisada por Klöppel et al. em $2004^{17}$.

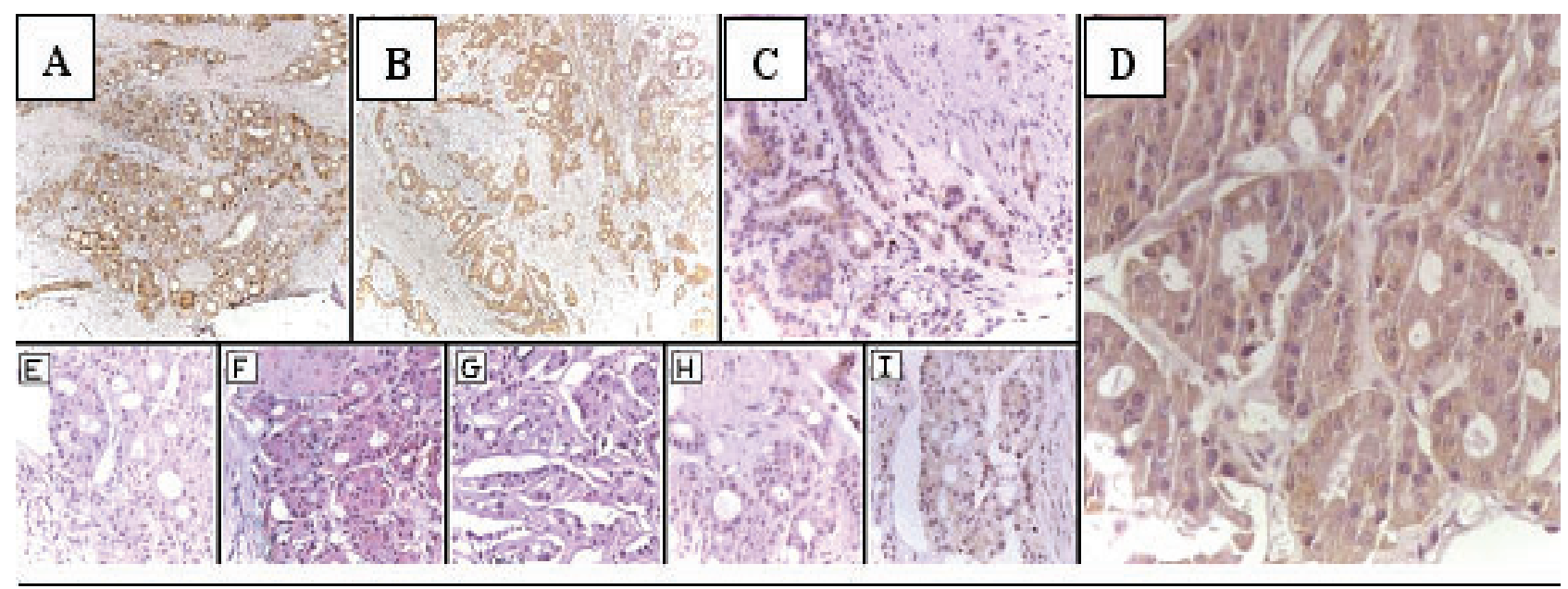

Figura 5. Imunohistoquímica do tumor na papila maior, onde se observa positividade para cromogranina (A); e sinaptofisina (B); e alguma positividade para Ki67 (C); nota-se uma evidente positividade global para somatostatina (D); e ausência de coloração para insulina $(E)$; glucagon $(F)$; gastrina $(G)$; peptídeo pancreático $(H)$; e serotonina (I). Este perfil fecha o diagnóstico para somatostatinoma. O tumor da papila menor apresentou perfil imunohistoquímico idêntico 
Sartorelli Jr AA et al. Somatostatinoma de papila maior e menor associado a tumor.

Tabela 2. Classificação dos tumores neuroendócrinos pela WHO e nomenclatura segundo Klöppel et al. ${ }^{17}$

\section{Subclasse 1}

Benigno: não funcionante, confinado à mucosa e submucosa, sem invasão vascular, $\leq 1 \mathrm{~cm}$.

Baixo grau de malignidade: confinado à mucosa e submucosa, com ou sem invasão vascular, $>1 \mathrm{~cm}$.

WHO: Tumor neuroendócrino

Klöppel: Tumor neuroendócrino

bem diferenciado (carcinóide)

\section{Subclasse 2}

Moderado grau de malignidade: invasão da muscular própria ou metástase

WHO: Carcinoma neuroendócrino

Klöppel: Carcinoma neuroendócrino

bem diferenciado (carcinóide maligno)

\section{Subclasse 3}

Alto grau de malignidade: carcinoma pouco diferenciado.

WHO: Carcinoma neuroendócrino mal

diferenciado

Klöppel: Carcinoma neuroendócrino

mal diferenciado (carcinóide maligno

de alto grau)

\section{DISCUSSÃO}

Os tumores periampulares são aqueles que se situam a até $1 \mathrm{~cm}$ de distância da papila de Vater, podendo se localizar na ampola, pâncreas, ducto biliar ou duodeno ${ }^{20,37}$. Os tumores primários de papila são considerados raros, com uma incidência aproximada de 6 casos por milhão, e apresentam diagnóstico mais precoce devido à sintomatologia de icterícia obstrutiva $^{13}$. Estes são mais freqüentes na papila maior do que na menor, e a coexistência de neoplasia em ambas as papilas é raramente descrita na literatura ${ }^{29}$.

Neste relato de caso, a microscopia das papilas pôde identificar interposição de tecido normal entre as áreas comprometidas pelas células neoplásicas, sendo este um achado indicativo de dois tumores primários (Figura 6). O perfil imunohistoquímico de positividade para sinaptofisina e cromogranina nestas lesões fechou o diagnóstico para tumores neuroendócrinos. Testou-se coloração para os diversos hormônios pancreáticos, ocorrendo somente positividade para somatostatina. Esta apresentação simultânea de somatostatinoma nas duas papilas é extremamente incomum, havendo apenas quatro casos descritos $^{4,5,12,34}$. Este é o subtipo mais raro dos tumores neuroendócrinos. A idade média do diagnóstico situa-se em torno de 50 anos, sendo igualmente encontrado em homens e mulheres ${ }^{11}$. Geralmente são malignos e de crescimento lento. Costumam apresentar sintomas em fase avançada, sendo que aproximadamente $75 \%$ dos pacientes possuem metástase no momento do diagnóstico ${ }^{38,44}$. Isto pode explicar as baixas taxas de sobrevida média observadas, que giram em torno de $30 \%$ em 2 anos, e apenas 15\% em 5 anos $^{10}$. Entretanto, conforme mencionado anteriormente, há indícios de uma maior sobrevida na apresentação periampular destes tumores. Atualmente, o tratamento de primeira escolha para estes tumores é cirúrgico, mesmo na presença de metástases, pois a quimioterapia é pouco efetiva para estas neoplasias ${ }^{2,8,44}$. Bettini et al. ${ }^{4}$ descreveram um paciente masculino com 47 anos, caucasiano, que apresentou dois somatostatinomas primários em ambas as papilas, associados com nódulos de GIST jejunal. Ele era portador da síndrome de Von Recklinghausen (NF1), e possuía diversas metástases hepáticas ao diagnóstico. Optou-se pela realização de uma duodenopancreatectomia com linfadenectomia extensa e ressecção de todas as massas tumorais, inclusive das metástases. Este paciente está atualmente com 24 meses sem recorrência da doença.

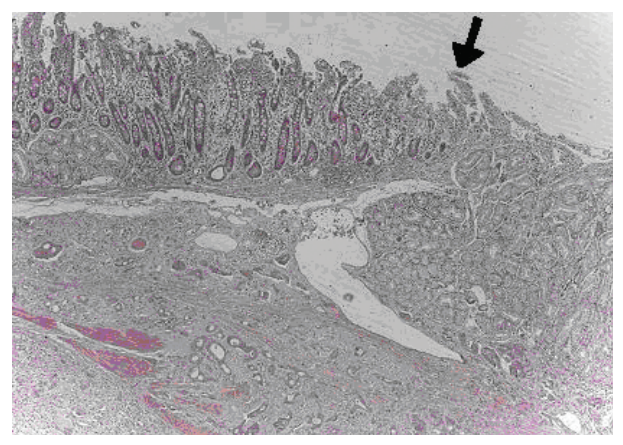

Figura 6. Seta mostra zona de transição entre epitélio colunar normal entre as papilas (à esquerda) e células neoplásicas em uma das papilas (à direita). 
Os tumores do estroma gastrointestinal (GIST) são derivados das células intersticiais de Cajal, responsáveis pelo peristaltismo ${ }^{32,42}$. São divididos em 3 tipos histológicos: a) epitelióides, que apresentam células globosas com citoplasma claro; b) fusocelular, com células de aspecto fasciculado ou neuróide, tendo um estroma mixóide ou muito vascularizado; c) Padrão misto. A característica imunohistoquímica que melhor define o GIST é a positividade para o receptor de tirosino-quinase CD117 (95\% dos casos), o qual está relacionado ao protooncogene KIT (c-kit). Isto os diferencia dos leiomiomas e dos outros tumores de músculo liso ${ }^{22,35}$. Também se pode encontrar concomitância de positividade para CD34 (70 a $80 \%$ ), actina de músculo liso (30 a $40 \%$ ) e, mais raramente, desmina e proteína $\mathrm{S}-100$ (menos de $5 \%)^{9,31}$. O comportamento biológico destes tumores é extremamente diversificado ${ }^{21}$. Em aproximadamente $50 \%$ dos casos há recorrência do tumor em 5 anos após uma ressecção completa ${ }^{24,39}$. Acredita-se que os principais determinantes de malignidade destes tumores são tamanho maior do que $5 \mathrm{~cm}$ e índice mitótico maior que 5 mitoses em 50 campos de grande aumento, apesar de existirem controvérsias sobre estes critérios ${ }^{3,23}$. A paciente deste caso apresentou nove nódulos jejunais com padrão imunohistoquímico positivo para c-kit, fechando diagnóstico para tumor do estroma gastrointestinal. A microscopia revelou um padrão fusocelular. Conforme mencionado anteriormente, os quatro casos relatados na literatura de pacientes com GIST e somatostatinoma são portadores de NF1. Se considerarmos que esta doença costuma manifestar-se nas duas primeiras décadas de vida, fica praticamente excluída a possibilidade de a paciente ser portadora ainda assintomática da síndrome.

O tratamento cirúrgico é a única opção de cura para os tumores do estroma gastrointestinal, deven- do-se tentar a ressecção total das lesões, inclusive das metástases ${ }^{1,27}$. Nesta paciente, quatro nódulos de GIST foram ressecados com a peça cirúrgica, e cinco nódulos separadamente. Foi realizada uma duodenopancreatectomia, a qual permanece amplamente apontada na literatura como conduta de escoIha para as neoplasias periampulare ${ }^{20,26,41}$, inclusive nos tumores neuroendócrinos ${ }^{4,30}$. Não é indicada uma ressecção linfonodal estendida para estes tumores, pois não existem evidências na literatura de melhora do prognóstico, e nos casos de adenocarcinomas ampulares, as evidências apontam não existir vantagem nesta ressecção ${ }^{15,19}$. Para reconstrução do trânsito intestinal, existe grande controvérsia nas vantagens e desvantagens da preservação pilórica. Dois importantes estudos de revisão e meta-análise recentes sugerem uma equivalência de resultados nos dois tipos de reconstrução, que consistem na técnica de Whipple clássica (ressecção pilórica com anastomose gastro-pancreática) e modificada (preservacão do piloro com anastomose pancreato-jejunal) ${ }^{7,18,43}$. $\mathrm{Na}$ preservação pilórica pode ocorrer gastroparesia no pós-operatório, sendo esta uma complicação importante. Porém, ela é auto-limitada, e os estudos apontam para uma melhora na qualidade de vida com a preservação do piloro gástrico ${ }^{7,18}$.

Muitas questões permanecem sem resposta, devido à ausência de casuística na literatura, principalmente quanto ao prognóstico e à sobrevida esperada para este caso. Apesar da metástase linfonodal proveniente dos carcinomas neuroendócrinos, as características das neoplasias sugerem bom prognóstico, tanto dos GIST como dos somatostatinomas. Infelizmente, existem poucos casos similares para serem tomados como referência. Trata-se de uma paciente não portadora de neurofibromatose, o que configura uma apresentação de tumores simultâneos totalmente inédita na literatura.

Sartorelli Jr, AA, Santana JAB, Pereira MFGO, Jukemura J, Cunha JEM, Assunção Neto RN. Somatostatinoma of the papilla major and minor associated with gastrointestinal stromal tumor (GIST). Rev Med (São Paulo). 2008 jan.-mar.;87(1):58-65.

\begin{abstract}
Somatostatinomas are very rare neuroendocrine tumors that have imunohistochemic positivity for somatostatin. The incidence of these tumors is higher in patients with the Von Recklinghausen's syndrome. There are four cases in the literature that show coexistence between somatostatinoma and gastrointestinal stromal tumors (GIST), all patients with neurofibromatosis. In this report we describe a non syndromic patient who had two primary somatostatinomas of both major and minor papillae, and nine jejunal GIST nodules. The diagnosis was confirmed by the anatomopathology and imunohistochemistry, and the treatment was duodenopancreatectomy and resection of the jejunal nodules. After the operation, the patient developed delayed gastric emptying. She was released from the Hospital in the $34^{\text {th }}$ day, with oral feeding reestablished. This is the first case reported in the literature that describes a synchronic appearance of these tumors in a patient that does not have neurofibromatosis.
\end{abstract}

KEY WORDS: Somatostatinoma Gastrointestinal Stromal Tumors/surgery. Ampulla of Vater /surgery. Immunohistochemistry 


\section{REFERÊNCIAS}

1. Aparicio T, Boige V, Sabourin JC, Crenn P, Ducreux $M$, Le Cesne A, et al. Prognostic factors after surgery of primary resectable gastrointestinal stromal tumours. Eur J Surg Oncol. 2004;30(10):1098-103.

2. Azimuddin $\mathrm{K}$, Chamberlain RS. The surgical management of pancreatic neuroendocrine tumors. Surg Clin North Am. 2001;81(3):511-25.

3. Bertin M, Angriman I, Scarpa M, Mencarelli R, Ranzato $\mathrm{R}$, et al. Prognosis of gastrointestinal stromal tumors. Hepatogastroenterology. 2007;54(73):124-8.

4. Bettini R, Falconi M, Crippa S, Capelli P, Boninsegna L, Pederzoli P. Ampullary somatostatinomas and jejunal gastrointestinal stromal tumor in a patient with Von Recklinghausen's disease. World J Gastroenterol. 2007;13(19):2761-3.

5. Borobia FG, Fabregat J, Jorba R, Poves I, Biondo $\mathrm{S}$, Serrano $\mathrm{T}$, et al. Exocrine pancreatic insufficiency caused by a somatostatinoma of the minor and major duodenal papilla in a patient with neurofibromatosis. Eur J Surg. 2001;167(2):154-6.

6. Dayal Y. Neuroendocrine tumors of the gastrointestinal tract. Pathol Case Rev. 2006;11(6):268-81.

7. Diener MK, Knaebel HP, Heukaufer C, Antes G, Büchler MW, Seiler CM. A systematic review and meta-analysis of pylorus-preserving versus classical pancreaticoduodenectomy for surgical treatment of periampullary and pancreatic carcinoma. Ann Surg. 2007;245(2):187-200.

8. Doherty GM. Rare endocrine tumours of the GI tract. Best Pract Res Clin Gastroenterol. 2005;19(5):80717.

9. Fletcher CD, Berman JJ, Corless C, Gorstein F, Lasota $\mathrm{J}$, Longley BJ, et al. Diagnosis of gastrointestinal stromal tumors: A consensus approach. Hum Pathol. 2002;33(5):459-65.

10. Hamy A, Heymann MF, Bodic J, Visset J, Le Borgne J, Leneel JC, et al. Duodenal somatostatinoma. anatomic/clinical study of 12 operated cases. Ann Chir. 2001;126(3):221-6.

11. Harris GJ, Tio F, Cruz AB Jr. Somatostatinoma: a case report and review of the literature. J Surg Oncol. 1987;36(1):8-16.

12. Heidt H, Hogel B, Giedl J, Gall FP, Ottenjann R. Malignant somatostatinoma of the papilla major and minor. Dtsch Med Wochenschr. 1989;114(45):1740-3.

13. Jean M, Dua K. Tumors of the ampulla of Vater. Curr Gastroenterol Rep. 2003;5(2):171-5.

14. Juergens KU, Weckesser M, Bettendorf O, Wormanns D. Duodenal somatostatinoma and gastrointestinal stromal tumor associated with neurofibromatosis type 1: diagnosis with PET/CT. AJR Am J Roentgenol. 2006;187(2):W233-4.

15. Kennedy EP, Yeo CJ. Pancreaticoduodenectomy with extended retroperitoneal lymphadenectomy for periampullary adenocarcinoma. Surg Oncol Clin N Am. 2007;16(1):157-76.

16. Klöppel G, Anlauf M. Pancreatic endocrine tumors. Pathol Case Rev. 2006;11(6):256-67.

17. Klöppel G, Perren A, Heitz PU. The gastroenteropancreatic neuroendocrine cell system and its tumors: the WHO classification. Ann N Y Acad Sci. 2004;1014:13-27.

18. McKay A, Mackenzie S, Sutherland FR, Bathe OF, Doig C, Dort J, et al. Meta-analysis of pancreaticojejunostomy versus pancreaticogastrostomy reconstruction after pancreaticoduodenectomy. Br J Surg. 2006;93:92936.

19. Michalski CW, Kleeff J, Wente MN, Diener MK, Büchler MW, Friess $\mathrm{H}$. Systematic review and meta-analysis of standard and extended lymphadenectomy in pancreaticoduodenectomy for pancreatic cancer. $\mathrm{Br} \mathrm{J}$ Surg. 2007;94(3):265-73.

20. Michelassi F, Erroi F, Dawson PJ, Pietrabissa A, Noda S, Handcock M, et al. Experience with 647 consecutive tumors of the duodenum, ampulla, head of the pancreas, and distal common bile duct. Ann Surg. 1989;210(4):544-54.

21. Miettinen M, Lasota J. Gastrointestinal stromal tumors: pathology and prognosis at different sites. Semin Diagn Pathol. 2006;23(2):70-83.

22. Miettinen M, Lasota J. Gastrointestinal stromal tumors - definition, clinical, histological, immunohistochemical, and molecular genetic features and differential diagnosis. Virchows Arch. 2001;438(1):1-12.

23. Miettinen M, Majidi M, Lasota J. Pathology and diagnostic criteria of gastrointestinal stromal tumors (GISTs): a review. Eur J Cancer. 2002;38(5):39-51.

24. Miettinen M, Sarlomo-Rikala M, Sobin LH, Lasota J. Gastrointestinal stromal tumors and leiomyosarcomas in the colon: a clinicopathologic, immunohistochemical, and molecular genetic study of 44 cases. Am J Surg Pathol. 2000;24(10):1339-52.

25. Mullan MH, Gauger PG, Thompson NW. Endocrine tumours of the pancreas: review and recent advances. ANZ J Surg. 2001;71(8):475-82.

26. Neoptolemos JP, Talbot IC, Shaw DC, Carr-Locke DL. Long-term survival after resection of ampullary carcinoma is associated independently with tumor grade and a new staging classification that assesses local invasiveness. Cancer. 1988;61(7):1403-7.

27. Ng EH, Pollock RE, Munsell MF, Atkinson EN, Romsdahl MM. Prognostic factors influencing survival in gastrointestinal leiomyosarcomas. Implications for surgical management and staging. Ann Surg. 1992;215(1):68-77.

28. Oberg K. Biology, diagnosis, and treatment of neuroendocrine tumors of the gastrointestinal tract. Curr Opin Oncol. 1994;6(4):441-51. 
29. Poultsides GA, Frederick WA. Carcinoid of the ampulla of Vater: morphologic features and clinical implications. World J Gastroenterol. 2006;12(43):7058-60.

30. Ricci JL. Carcinoid of the ampulla of vater. Local resection or pancreaticoduodenectomy. Cancer. 1993;71(3):686-90.

31. Rubin BP, Fletcher JA, Fletcher CD. Molecular insights into the histogenesis and pathogenesis of gastrointestinal stromal tumors. Int J Surg Pathol. 2000;8(1):5-10.

32. Sircar K, Hewlett BR, Huizinga JD, Chorneyko K, Berezin I, Riddell RH. Interstitial cells of Cajal as precursors of gastrointestinal stromal tumors. Am J Surg Pathol. 1999;23(4):377-89.

33. Soga J, Yakuwa Y. Somatostatinoma/inhibitory syndrome: a statistical evaluation of 173 reported cases as compared to other pancreatic endocrinomas. J Exp Clin Cancer Res. 1999;18(1):13-22.

34. Stommer PE, Stolte M, Seifert E. Somatostatinoma of vater's papilla and of the minor papilla. Cancer. 1987;60(2):232-5.

35. Susan CA. Distinguishing gastrointestinal stromal tumors from their mimics: an update. Adv Anat Pathol. 2007; 14:178-88

36. Suzuki S, Sato K, Katada E, Kuno Y, Mizoguchi N, Tokuda $\mathrm{H}$, et al. Periampullary somatostatinoma and multiple gastrointestinal stromal tumors associated with von Recklinghausen's disease. J Gastroenterol. 2004;39(10):1011-2.

37. Talamini MA, Moesinger RC, Pitt HA, et al. Adenocarcinoma of the ampulla of vater: a 28-year experience. Ann Surg. 1997;225:590-600.
38. Tanaka S, Yamasaki S, Matsushita H, Ozawa Y, Kurosaki A, Takeuchi K, et al. Duodenal somatostatinoma: a case report and review of 31 cases with special reference to the relationship between tumor size and metastasis. Pathol Int. 2000;50(2):146-52.

39. Tzen CY, Wang JH, Huang YJ, Wang MN, Lin PC, Lai $\mathrm{GL}$, et al. Incidence of gastrointestinal stromal tumor: a retrospective study based on immunohistochemical and mutational analyses. Dig Dis Sci. 2007;52(3):792-7.

40. Usui M, Matsuda S, Suzuki H, Hirata K, Ogura Y, Shiraishi T. Somatostatinoma of the papilla of Vater with multiple gastrointestinal stromal tumors in a patient with von Recklinghausen's disease. J Gastroenterol. 2002;37(11):947-53.

41. Wagle PK, Joshi RM, Mathur SK. Pancreaticoduodenectomy for periampullary carcinoma. Indian. J Gastroenterol. 2001;20(2):53-5.

42. Wang L, Vargas H, French SW. Cellular origin of gastrointestinal stromal tumors: a study of 27 cases. Arch Pathol Lab Med. 2000;124(10):1471-5.

43. Wente MN, Shrikhande SV, Müller MW, Diener MK, Seiler $\mathrm{CM}$, Friess $\mathrm{H}$, Büchler MW. Pancreaticojejunostomy versus pancreaticogastrostomy: systematic review and meta-analysis. Am J Surg. 2007;193(2):171-83.

44. Wilson MC, Stephen PM, Grant S, Andrew ML. Ampullary carcinoid tumors: rationale for an aggressive surgical approach. J Gastrointest Surg. 2003;7:773-6.

45. Zhou Hui, Schaefer N, Wolff M, Fischer HP. Carcinoma of the Ampulla of Vater: comparative histologic/ Immunohistochemical classification and follow-up. Am J Surg Pathol. 2004;28(7):875-82. 